\title{
A Cost per Responder Model for Abatacept versus Adalimumab Among Rheumatoid Arthritis Patients with Seropositivity
}

This article was published in the following Dove Press journal:

ClinicoEconomics and Outcomes Research

\author{
Sang Hee Park' \\ Xue $\mathrm{Han}^{2}$ \\ Francis Lobo ${ }^{2}$ \\ Sakina Nanji \\ Dipen Patel $\mathbb{B}^{\prime}$
}

'Modeling and Meta-Analysis, Pharmerit International, Bethesda, MD, USA; ${ }^{2}$ WW HEOR Markets-US, Bristol-Myers Squibb Company, Lawrenceville, NJ, USA
Correspondence: Xue Han

Bristol-Myers Squibb Company, 340 I

Princeton Pike, Lawrenceville, NJ 08648,

USA

Tel + | 213-309-97|9

Fax +I 6093025808

Email Xue.Han I@bms.com
Purpose: The primary objective of this study was to compare the cost per responder (CPR) between abatacept and adalimumab among seropositive rheumatoid arthritis (RA) patients.

Patients and Methods: CPR analysis was conducted from a US payer perspective over 24 weeks for early moderate-to-severe seropositive RA patients. Efficacy data (American College of Rheumatology [ACR] improvement criteria [ACR20/50/70] and DAS28-C reactive protein $<2.6$ ) for abatacept and adalimumab were sourced from the post hoc analysis of the Early AMPLE trial (NCT02557100). Medication costs were considered assuming complete adherence. A 30\% rebate was applied for adalimumab in the base case.

Results: At week 24, the total per patient pharmacy cost was $\$ 26,273.34$ and $\$ 21,731.18$, whereas the CPR (using ACR70 as the responder definition) was $\$ 46,337.46$ and $\$ 74,935.10$ (difference of $-\$ 28,597.64$ ) for abatacept and adalimumab, respectively. The CPR was consistently lower for abatacept compared to adalimumab across all clinical measures, with differences ranging from $-\$ 7099.32$ to $-\$ 43,608.97$.

Conclusion: While the pharmacy cost was higher for abatacept compared to adalimumab, due to its higher clinical efficacy, the CPR was consistently lower for seropositive RA patients treated with abatacept. The results may be useful for healthcare decision-makers in understanding how to optimize treatment for seropositive RA patients while minimizing costs in today's budget-constrained health environment.

Keywords: positive shared epitope, anti-citrullinated protein antibodies, anti-cyclic citrullinated peptide, rheumatoid factor, biomarker, seropositive

\section{Introduction}

Rheumatoid arthritis (RA) is a chronic inflammatory disease, affecting approximately $0.5 \%$ of the United States (US) population. ${ }^{1}$ Given that it is a chronic disease, the primary objective of RA treatment is to achieve a state of clinical remission to prevent further irreversible damages. ${ }^{2}$ In the past three decades, the treatment options for RA patients have advanced substantially, and currently, there are multiple safe and effective treatments available. ${ }^{3}$ Tumor necrosis factors inhibitors (TNFis) were the first biologic disease-modifying anti-rheumatic drugs (bDMARDs) developed for RA and have been the most frequently prescribed class of bDMARDs for patients who fail conventional synthetic DMARDs (csDMARDs). Additional bDMARDs with different mechanisms of action such as interleukin 6 receptor inhibition, T-cell costimulation inhibition, and B-cell depletion have been developed as well as more recent synthetic targeted DMARDs. Despite the improved efficacy of treatments, 
however, drug discontinuation persists due to lack of efficacy or adverse events on the selected treatment. ${ }^{4-8}$ This leads to treatment switches which are associated with higher medical cost, lower efficacy, and reduced quality of life. ${ }^{4,9,10}$ Given such drawbacks, carefully selecting their initial treatment rather than a trial-and-error basis to reduce switching is critical.

While biomarkers have not been used widely to guide treatment selection in this therapeutic area, recent studies have shown that patients with positive shared epitope ( $\mathrm{SE}+$ ), a five amino acid sequence motif in residues 7074 of the HLADR $\beta 1$ chain that has been associated with severe cases of RA, ${ }^{11-14}$ respond well to abatacept and this may be an objective marker for treatment selection. A post hoc analysis of the Early AMPLE study, a phase 4 single-blinded randomized trial evaluating the efficacy of the subcutaneous (SC) form of abatacept in early RA ( $\leq 12$ months since the first diagnosis) patients with moderate-to-high disease activity and positive anti-citrullinated protein antibodies $(\mathrm{ACPA}+)$ and positive rheumatoid factor $(\mathrm{RF}+)$, showed that $\mathrm{SE}+$ patients treated with abatacept responded well at week 24 across American College of Rheumatology (ACR) 20\%, 50\%, and 70\% improvement criteria (ACR20, ACR50, ACR70) and Disease Activity Score 28-joint count $\mathrm{C}$ reactive protein (DAS28-CRP) $<2.6{ }^{15}$ Additionally, Oryoji et al retrospectively evaluated 72 Japanese patients (45 SE+ and 25 SE-) treated with abatacept and found that the Simple Disease Activity Index (SDAI) remission was higher among $\mathrm{SE}+$ patients at week $24(55.3 \%$ vs $20.0 \%, \mathrm{p}=0.01)$ and the SE status was an independent predictor of SDAI remission after adjusting for ACPA titer, age, sex, SDAI at baseline, methotrexate use, and prior use of biological agents. ${ }^{16}$

Despite the illustrated efficacy of abatacept among seropositive patients, economic assessments of abatacept compared with adalimumab and other TNFis are currently unavailable. To address this gap for economic evaluation, we developed a cost per responder (CPR) model to compare CPR between abatacept SC and adalimumab SC from a US payer perspective among early moderate-to-severe RA patients with seropositivity ( $\mathrm{SE}+, \mathrm{ACPA}+$, and $\mathrm{RF}+$ ) using the results from the post hoc analysis of the Early AMPLE trial. ${ }^{15}$

\section{Methods}

\section{Model Structure}

The CPR analysis was structured as a simple economic assessment programmed in Microsoft Excel. The analysis was from a hypothetical US commercial payer perspective over 24 weeks and the target population was early moderate-tosevere RA patients with seropositivity (SE+, ACPA+, and $\mathrm{RF}+$ ). The two treatment options compared in the base case were abatacept SC and adalimumab SC.

The total cost for each treatment option was calculated as the treatment cost over 24 weeks for the patient and the CPR was calculated as the total treatment cost divided by the efficacy (ie, proportion of responders). Additionally, the difference in CPR was calculated by taking the difference in the CPRs between the two treatment options.

\section{Model Parameters \\ Patient Weight}

The default average weight of a patient was assumed to be $80.4 \mathrm{~kg}$, calculated as the weighted average of US female and male patient weight, with gender distribution sourced from an RA study. ${ }^{1,17}$

\section{Efficacy}

Responder was defined as those patients who have achieved the specified efficacy outcomes (ACR20, ACR50, ACR70, and DAS 28-CRP <2.6) at week 24. Clinical efficacy data were based on the post hoc analysis of the 24-week results of the phase 4 head-to-head Early AMPLE trial (NCT02557100); ${ }^{15}$ the results for SE+ patients who received abatacept $(n=30)$ and those who received adalimumab $(n=31)$ were digitized to generate the values used in our analysis (Table 1). ${ }^{15}$

\section{Medication Costs}

The wholesale acquisition cost (WAC) for each medication in 2019 US dollars was used for medication costs. ${ }^{18}$ The prescribing information was referenced to estimate the number of doses needed for patients newly initiating each drug over a 24-week course, assuming no vial sharing and complete adherence to the medication. Medications

Table I Efficacy Data by Treatment ${ }^{15}$

\begin{tabular}{|l|l|l|l|l|}
\hline Drug Name & ACR20 & ACR50 & ACR70 & $\begin{array}{l}\text { DAS28- } \\
\text { CRP <2.6 }\end{array}$ \\
\hline Abatacept & $86.7 \%$ & $76.7 \%$ & $56.7 \%$ & $50.0 \%$ \\
Adalimumab & $58.1 \%$ & $45.2 \%$ & $29.0 \%$ & $22.6 \%$ \\
\hline
\end{tabular}

Note: Table created with data from Park SH, Han X, Lobo F, Nanji S, Patel D. A cost per responder analysis of abatacept versus adalimumab for the treatment of rheumatoid arthritis among patients with shared epitope (SE) positivity from a United States payer perspective. Ann Rheum Dis. 2020;79(Suppl I):1872-1873. ${ }^{26}$

Abbreviations: ACR20/50/70, American College of Rheumatology 20\%, 50\%, 70\% improvement criteria; DAS28-CRP, Disease Activity Score 28 -joint count C reactive protein. 
Table 2 Medication Costs (2019 US Dollars)

\begin{tabular}{|c|c|c|c|c|c|c|}
\hline Drug Name & Dose $^{a}$ & Dose Frequency ${ }^{a}$ & Strength & Package Size & Packages per 24 Weeks & $\operatorname{WAC}(\$)^{18, \mathrm{~b}}$ \\
\hline Abatacept SC & $125 \mathrm{mg} / \mathrm{mL}$ & Once weekly & $125 \mathrm{mg}$ & 4 & 6 & $\$ 4378.89$ \\
\hline Adalimumab SC & $40 \mathrm{mg}$ & Every 2 weeks & $40 \mathrm{mg}$ & 2 & 6 & $\$ 5174.09$ \\
\hline
\end{tabular}

Notes: ${ }^{a}$ Dose and dose frequency information from each product's package insert. ${ }^{\text {t}}$ The cost does not reflect any rebates.

Abbreviations: SC, subcutaneous; US, United States; WAC, wholesale acquisition cost.

cost and prescribing information for each drug are presented in Table 2.

\section{Scenario Analyses \\ Rebates}

In the base case scenario, a $30 \%$ rebate was applied for adalimumab to reflect real-world pricing in the US Additionally, we conducted the following scenario analyses: 1) no rebates for any medication and 2) $15 \%$ rebate for abatacept with $30 \%$ rebate for adalimumab.

\section{Forty-Eight Weeks}

We conducted a scenario analysis for CPR at 48 weeks. Efficacy data were based on the post hoc analysis of the 24-week results of the Early AMPLE trial and were assumed to be the same at week $48 .{ }^{15}$

\section{Results}

At week 24, the total per patient pharmacy cost was $\$ 26,273.34$ for abatacept and $\$ 21,731.18$ for adalimumab (difference: \$4542.16) in the base case. The CPR (using
ACR70 as the responder definition) was $\$ 46,337.46$ for abatacept and $\$ 74,935.10$ for adalimumab (difference: $-\$ 28,597.64$; Figure 1). The CPR was consistently lower for abatacept compared to adalimumab across all clinical measures, with differences ranging from $-\$ 7099.32$ to $-\$ 43,608.97$. With no rebates, total pharmacy cost at week 24 was $\$ 4771.20$ lower for abatacept compared to adalimumab and the difference in CPR was even greater (Table 3). With a $15 \%$ rebate for abatacept and a $30 \%$ rebate for adalimumab, pharmacy cost per patient was higher by $\$ 601.16$ for abatacept but the CPR was continuously lower for abatacept (Table 4). In the 48-week scenario analysis with a $30 \%$ rebate for adalimumab, pharmacy cost per patient was $\$ 9084.32$ higher for abatacept compared to adalimumab, but the CPR was consistently lower for abatacept across all clinical measures (Table 5).

\section{Discussion}

With many RA treatment options being available and high treatment costs, evaluating the comparative treatment costs is

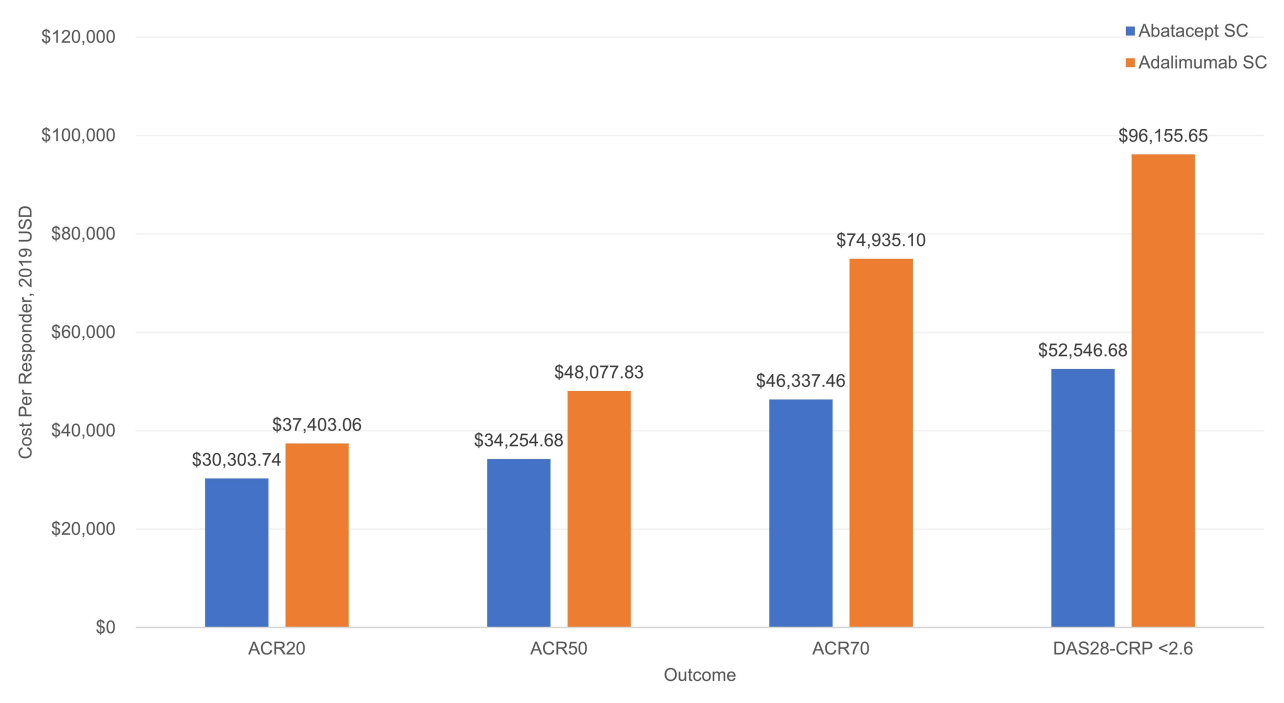

Figure I Base case results: cost per responder, $30 \%$ rebate for adalimumab.

Note: Figure created with data from Park SH, Han X, Lobo F, Nanji S, Patel D. A cost per responder analysis of abatacept versus adalimumab for the treatment of rheumatoid arthritis among patients with shared epitope (SE) positivity from a United States payer perspective. Ann Rheum Dis. 2020;79(Suppl I):1872-1873. ${ }^{26}$

Abbreviations: ACR20/50/70, American College of Rheumatology 20\%, 50\%, 70\% improvement criteria; DAS28-CRP, Disease Activity Score 28-joint count C reactive protein; SC, subcutaneous; USD, US dollars. 
Table 3 Scenario Analysis Results: No Rebates

\begin{tabular}{|l|l|l|l|}
\hline Outcome & Abatacept SC & Adalimumab SC & Difference \\
\hline Pharmacy Cost Per Patient & $\$ 26,273.34$ & $\$ 31,044.54$ & $-\$ 4771.20$ \\
Cost Per Responder - ACR20 & $\$ 30,303.74$ & $\$ 53,432.94$ & $-\$ 23,129.21$ \\
Cost Per Responder - ACR50 & $\$ 34,254.68$ & $\$ 68,682.61$ & $-\$ 34,427.93$ \\
Cost Per Responder - ACR70 & $\$ 46,337.46$ & $\$ 107,050.14$ & $-\$ 60,712.68$ \\
Cost Per Responder - DAS28-CRP <2.6 & $\$ 52,546.68$ & $\$ 137,365.22$ & $-\$ 84,818.54$ \\
\hline
\end{tabular}

Abbreviations: ACR20/50/70, American College of Rheumatology 20\%, 50\%, 70\% improvement criteria; DAS28-CRP, Disease Activity Score 28 -joint count C reactive protein; SC, subcutaneous.

Table 4 Scenario Analysis Results: 15\% Rebate for Abatacept and 30\% for Adalimumab

\begin{tabular}{|l|l|l|l|}
\hline Outcome & Abatacept SC & Adalimumab SC & Difference \\
\hline Pharmacy Cost Per Patient & $\$ 22,332.34$ & $\$ 21,731.18$ & $\$ 601.16$ \\
Cost Per Responder - ACR20 & $\$ 25,758.18$ & $\$ 37,403.06$ & $-\$ 11,644.88$ \\
Cost Per Responder - ACR50 & $\$ 29,116.48$ & $\$ 48,077.83$ & $-\$ 18,961.35$ \\
Cost Per Responder - ACR70 & $\$ 39,386.84$ & $\$ 74,935.10$ & $-\$ 35,548.26$ \\
Cost Per Responder - DAS28-CRP <2.6 & $\$ 44,664.68$ & $\$ 96,155.65$ & $-\$ 51,490.98$ \\
\hline
\end{tabular}

Abbreviations: ACR20/50/70, American College of Rheumatology 20\%, 50\%, 70\% improvement criteria; DAS28-CRP, Disease Activity Score 28-joint count C reactive protein; SC, subcutaneous.

Table 5 Scenario Analysis Results: 30\% Rebate for Adalimumab at 48 Weeks

\begin{tabular}{|l|l|l|l|}
\hline Outcome & Abatacept SC & Adalimumab SC & Difference \\
\hline Pharmacy Cost Per Patient & $\$ 52,546.68$ & $\$ 43,462.36$ & $\$ 9084.32$ \\
Cost Per Responder - ACR20 & $\$ 60,607.47$ & $\$ 74,806.12$ & $-\$ 14,198.65$ \\
Cost Per Responder - ACR50 & $\$ 68,509.36$ & $\$ 96,155.65$ & $-\$ 27,646.29$ \\
Cost Per Responder - ACR70 & $\$ 92,674.92$ & $\$ 149,870.19$ & $-\$ 57,195.27$ \\
Cost Per Responder - DAS28-CRP <2.6 & $\$ 105,093.36$ & $\$ 192,311.31$ & $-\$ 87,217.95$ \\
\hline
\end{tabular}

Abbreviations: ACR20/50/70, American College of Rheumatology 20\%, 50\%, 70\% improvement criteria; DAS28-CRP, Disease Activity Score 28 -joint count C reactive protein; SC, subcutaneous.

important for decision-makers. The CPR analysis is useful as it combines both clinical and economic dimensions. While the exact prevalence of the target population is unknown, it is estimated that only up to $20 \%$ of RA patients are SE- and $75.9 \%$ of RA patients are ACPA+ and among those ACPA+ patients, $90.1 \%$ of patients are $\mathrm{RF}+{ }^{12,20}$ With an estimated large target population, it is important to consider CPR in treatment decision-making, as incorporating treatments with low CPR can result in efficient healthcare spending. Our results showed that due to the increased efficacy of abatacept among seropositive patients, abatacept has a lower CPR among seropositive patients compared to adalimumab. Given the lack of randomized clinical trial efficacy data for other TNFis in seropositive RA patients, if the efficacy of other TNFis were assumed to be the same as adalimumab (ie, the only head-to-head comparison against abatacept yet available in this population), CPRs for other TNFis (using ACR70 as responder definition) could range from $\$ 51,946.72$ to $\$ 223,832.59$, depending on product costs.
To our knowledge, this is the first economic analysis for RA patients with $\mathrm{SE}+$ as well as ACPA+ and RF+. This is important because recent studies have found that $\mathrm{SE}+$ is an independent predictor of SDAI remission apart from ACPA titer, ${ }^{16}$ so economic analyses for this biomarker can help in decision-making. Previous economic assessment studies using data from the phase 3 AMPLE trial (NCT00929864) looked into RA biomarkers including RF and ACPA but did not include SE. Foo and colleagues studied the cost per additional health gain (ie, patient response or patient in remission) between abatacept and adalimumab among two cohorts: Cohort 1 included patients with early RA, RF+ and/or ACPA+, and $>1$ radiographic erosion; Cohort 2 included RA patients with at least one of the previous criteria not being met. ${ }^{21}$ The model showed that abatacept was favored in all countries studied (US, Germany, Spain, and Canada) except for DAS28-CRP remission in Canada. The study 
found that cost savings were greater with the more stringent Cohort 1 than Cohort 2. Weijers and colleagues evaluated the cost per responder or remission by ACPA status for patients receiving abatacept and adalimumab. ${ }^{22}$ The study found that in Spain and Germany, abatacept always had lower CPR for ACPA+ patients across all definitions used for responder/remission (ie, ACR20, ACR50, ACR70, ACR90, Health Assessment Questionnaire-Disability Index, DAS28-CRP, Clinical Disease Activity Index, and SDAI). For Italy, Canada, and the US, abatacept was again favored for ACPA+ patients, except for ACR50 response and DAS28-CRP remission. Both studies highlight that the economic efficiency of abatacept is even more pronounced among patients with certain clinical biomarkers. These two CPR studies focused on RF and/or ACPA in the AMPLE trial and did not include SE, but given that the concentrations of ACPA and SE are correlated, ${ }^{23,24}$ the consistent results that abatacept is a cost-efficient treatment choice among the studied patients in the AMPLE trial are not surprising and are congruous with our results for abatacept in SE+ patients in the Early AMPLE trial. However, when comparing these CPR studies to our results, it is worth noting that the phase 3 AMPLE trial was not designed to estimate the efficacy of abatacept SC versus adalimumab SC in ACPA subgroups and may lack power to estimate significant differences between these subgroups. ${ }^{22}$ In contrast, our analysis is based on results from the phase 4 Early AMPLE trial, ${ }^{15}$ which includes changes in seropositivity as a primary outcome measure. ${ }^{25}$

With more evidence pointing towards the need for personalized medicine in RA based on biomarkers and serology, our study adds the economic evidence among the subpopulation of $\mathrm{SE}+$, ACPA+, and $\mathrm{RF}+$ patients. However, the findings from our study are subject to several limitations. First, concomitant medications and drug monitoring tests are not included in the analysis, yet these costs are typically low and similar enough that the results are expected to remain the same. Next, not all parameters included in the analysis, such as the rebate rates, are generalizable and vary by health plans. Therefore, scenario analyses on rebates were conducted, which showed that the CPR was consistently lower for abatacept. Finally, in the 48-week scenario analysis, the efficacy inputs at week 48 were based on Early AMPLE trial data at week 24, due to the lack of 48 -week data availability in the Early AMPLE trial.

\section{Conclusion}

While the pharmacy cost in our analysis was higher for abatacept compared to adalimumab, due to its higher clinical efficacy, the CPR was consistently lower for seropositive RA patients treated with abatacept. The results may be useful for healthcare decision-makers in understanding how to optimize treatment for seropositive RA patients while minimizing costs in today's budget-constrained health environment.

\section{Abbreviations}

ACPA, anti-citrullinated protein antibodies; ACR, American College of Rheumatology; ACR20, ACR 20\% improvement criteria; ACR50, ACR 50\% improvement criteria; ACR70, ACR 70\% improvement criteria; ACR90, ACR 90\% improvement criteria; bDMARDs, biologic disease-modifying anti-rheumatic drugs; CPR, cost per responder; csDMARDs, conventional synthetic diseasemodifying anti-rheumatic drugs; DAS28-CRP, Disease Activity Score 28-joint count $\mathrm{C}$ reactive protein; RA, rheumatoid arthritis; RF, rheumatoid factor; SC, subcutaneous; SDAI, Simple Disease Activity Index; SE, shared epitope; TNFi, tumor necrosis factors inhibitor; US, United States; WAC, wholesale acquisition cost.

\section{Acknowledgments}

The study was funded by Bristol-Myers Squibb Company. The CPR results in this article were presented in part at as an abstract for the 2020 Annual European Congress of Rheumatology (EULAR) virtual meeting, June 2020.

\section{Disclosure}

$\mathrm{XH}$ and FL are employees of Bristol-Myers Squibb Company. SHP is a former employee of Pharmerit International and is currently an employee of Bristol-Myers Squibb Company. DP and SN are current employees of Pharmerit International, which received consultancy fees from Bristol-Myers Squibb Company for this study. The authors report no other conflicts of interest in this work.

\section{References}

1. Hunter TM, Boytsov NN, Zhang X, Schroeder K, Michaud K, Araujo AB. Prevalence of rheumatoid arthritis in the United States adult population in healthcare claims databases, 2004-2014. Rheumatol Int. 2017;37(9):1551-1557. doi:10.1007/s00296-0173726-1

2. Singh JA, Saag KG, Bridges SL Jr, et al. 2015 American College of Rheumatology guideline for the treatment of rheumatoid arthritis. Arthritis Rheumatol. 2016;68(1):1-26. doi:10.1002/art.39480 
3. Favalli EG, Raimondo MG, Becciolini A, Crotti C, Biggioggero M, Caporali R. The management of first-line biologic therapy failures in rheumatoid arthritis: current practice and future perspectives. Autoimmun Rev. 2017;16(12):1185-1195. doi:10.1016/j.autrev.2017. 10.002

4. Gu T, Mutebi A, Stolshek BS, Tan H. Cost of biologic treatment persistence or switching in rheumatoid arthritis. Am J Manag Care. 2018;24(8 Spec No.):SP338-SP345.

5. Nam JL, Winthrop KL, van Vollenhoven RF, et al. Current evidence for the management of rheumatoid arthritis with biological disease-modifying antirheumatic drugs: a systematic literature review informing the EULAR recommendations for the management of RA. Ann Rheum Dis. 2010;69(6):976-986. doi:10.1136/ $\operatorname{ard} .2009 .126573$

6. Marchesoni A, Zaccara E, Gorla R, et al. TNF-alpha antagonist survival rate in a cohort of rheumatoid arthritis patients observed under conditions of standard clinical practice. Ann N Y Acad Sci. 2009;1173(1):837-846. doi:10.1111/j.17496632.2009.04621.x

7. Favalli EG, Pregnolato F, Biggioggero M, et al. Twelve-year retention rate of first-line tumor necrosis factor inhibitors in rheumatoid arthritis: real-life data from a local registry. Arthritis Care Res (Hoboken). 2016;68(4):432-439. doi:10.1002/ acr.22788

8. Atzeni F, Sarzi-Puttini P, Botsios C, et al. Long-term anti-TNF therapy and the risk of serious infections in a cohort of patients with rheumatoid arthritis: comparison of adalimumab, etanercept and infliximab in the GISEA registry. Autoimmun Rev. 2012;12 (2):225-229. doi:10.1016/j.autrev.2012.06.008

9. Vanderpoel J, Tkacz J, Brady BL, Ellis L. Health care resource utilization and costs associated with switching biologics in rheumatoid arthritis. Clin Ther. 2019;41(6):1080-1089 e1085. doi:10.1016/j. clinthera.2019.04.032

10. Zhang J, Shan Y, Reed G, et al. Thresholds in disease activity for switching biologics in rheumatoid arthritis patients: experience from a large U.S. cohort. Arthritis Care Res (Hoboken). 2011;63 (12):1672-1679. doi:10.1002/acr.20643

11. Gregersen PK, Silver J, Winchester RJ. The shared epitope hypothesis. An approach to understanding the molecular genetics of susceptibility to rheumatoid arthritis. Arthritis Rheum. 1987;30 (11):1205-1213. doi:10.1002/art.1780301102

12. Holoshitz J. The rheumatoid arthritis HLA-DRB1 shared epitope. Curr Opin Rheumatol. 2010;22(3):293-298. doi:10.1097/BOR.0b013 e328336ba63

13. Gonzalez-Gay MA, Garcia-Porrua C, Hajeer AH. Influence of human leukocyte antigen-DRB1 on the susceptibility and severity of rheumatoid arthritis. Semin Arthritis Rheum. 2002;31(6):355-360. doi:10.1053/sarh.2002.32552

14. Turesson C, Schaid DJ, Weyand CM, et al. The impact of HLA-DRB1 genes on extra-articular disease manifestations in rheumatoid arthritis. Arthritis Res Ther. 2005;7(6):R1386-R1393. doi:10.1186/ar1837
15. Rigby W, Buckner J, Bridges L, et al. The effect of HLA-DRB1 risk alleles on the clinical efficacy of abatacept and adalimumab in seropositive biologic-naive patients with early, moderate-to-severe RA: data from a head-to-head single-blinded trial. Ann Rheum Dis. 2019;78(Suppl 2):263-264. doi:10.1136/annrheumdis-2019eular.8668

16. Oryoji K, Yoshida K, Kashiwado Y, et al. Shared epitope positivity is related to efficacy of abatacept in rheumatoid arthritis. Ann Rheum Dis. 2018;77(8):1234-1236. doi:10.1136/annrheumdis-2017-211430

17. Fryar CD, Kruszon-Moran D, Gu Q, Ogden CL. Mean body weight, height, waist circumference, and body mass index among adults: United States, 1999-2000 through 2015-2016. Natl Health Stat Report. 2018;(122):1-16.

18. Micromedex ${ }^{\circledR}$ (electronic version). Greenwood Village, Colorado, USA: IBM Watson Health. Available from: https://www.micromedex solutions.com. Accessed September 11, 2019.

19. CMS Physician Fee Schedule 2019. Available from: https://www. cms.gov/apps/physician-fee-schedule/search/search-criteria.aspx. Accessed September 11, 2019.

20. Bapat B, Klink A, Kaufman J, et al. ACPA testing and resultant treatment patterns in patients with rheumatoid arthritis: findings from US community rheumatology practices [abstract]. Arthritis Rheumatol. 2019;71(suppl 10).

21. Foo J, Morel C, Bergman M, et al. Cost per response for abatacept versus adalimumab in patients with seropositive, erosive early rheumatoid arthritis in the US, Germany, Spain, and Canada. Rheumatol Int. 2019;39(9):1621-1630. doi:10.1007/s00296-019-04352-2

22. Weijers L, Baerwald C, Mennini FS, et al. Cost per response for abatacept versus adalimumab in rheumatoid arthritis by ACPA subgroups in Germany, Italy, Spain, US and Canada. Rheumatol Int. 2017;37(7):1111-1123. doi:10.1007/s00296-017-3739-9

23. Klareskog L, Ronnelid J, Lundberg K, Padyukov L, Alfredsson L. Immunity to citrullinated proteins in rheumatoid arthritis. Annu Rev Immunol. 2008;26:651-675. doi:10.1146/annurev.immunol.26.02 1607.090244

24. Murphy D, Mattey D, Hutchinson D. Anti-citrullinated protein antibody positive rheumatoid arthritis is primarily determined by rheumatoid factor titre and the shared epitope rather than smoking per se. PLoS One. 2017;12(7):e0180655. doi:10.1371/journal.pone.0180655

25. ClinicalTrials.gov identifier: NCT02557100. Study to assess changes in the immune profile in adults with early rheumatoid arthritis; [updated November 27, 2019]. Available from: https://www.clinical trials.gov/ct2/show/study/NCT02557100. Accessed August 5, 2020.

26. Park SH, Han X, Lobo F, Nanji S, Patel D. A cost per responder analysis of abatacept versus adalimumab for the treatment of rheumatoid arthritis among patients with shared epitope (SE) positivity from a United States payer perspective. Ann Rheum Dis. 2020;79 (Suppl 1):1872-1873. doi:10.1136/annrheumdis-2020-eular.1019
ClinicoEconomics and Outcomes Research

\section{Publish your work in this journal}

ClinicoEconomics and Outcomes Research is an international, peerreviewed open-access journal focusing on Health Technology Assessment, Pharmacoeconomics and Outcomes Research in the areas of diagnosis, medical devices, and clinical, surgical and pharmacological intervention. The economic impact of health policy and health systems

Submit your manuscript here: https://www.dovepress.com/clinicoeconomics-and-outcomes-research-journa organization also constitute important areas of coverage. The manuscript management system is completely online and includes a very quick and fair peer-review system, which is all easy to use. Visit http://www.dovepress.com/testimonials.php to read real quotes from published authors. 